\title{
Spatial Clustering of Structured Objects
}

\author{
Donato Malerba, Annalisa Appice, Antonio Varlaro, and Antonietta Lanza \\ Dipartimento di Informatica, Università degli Studi di Bari, \\ via Orabona, 4 - 70126 Bari - Italy \\ \{malerba, appice, varlaro, lanza\}@di.uniba.it
}

\begin{abstract}
Clustering is a fundamental task in Spatial Data Mining where data consists of observations for a site (e.g. areal units) descriptive of one or more (spatial) primary units, possibly of different type, collected within the same site boundary. The goal is to group structured objects, i.e. data collected at different sites, such that data inside each cluster models the continuity of socio-economic or geographic environment, while separate clusters model variation over the space. Continuity is evaluated according to the spatial organization arising in data, namely discrete spatial structure, expressing the (spatial) relations between separate sites implicitly defined by their geometrical representation and positioning. Data collected within sites that are (transitively) connected in the discrete spatial structure are clustered together according to the similarity on multi-relational descriptions representing their internal structure. CORSO is a novel spatial data mining method that resorts to a multi-relational approach to learn relational spatial data and exploits the concept of neighborhood to capture relational constraints embedded in the discrete spatial structure. Relational data are expressed in a firstorder formalism and similarity among structured objects is computed as degree of matching with respect to a common generalization. The application to real-world spatial data is reported.
\end{abstract}

\section{Introduction}

Within both social and environmental sciences much of data is collected in a spatial framework, where data consists of measurements or observations of one or more attributes taken at specific sites which are spatially-referenced. This means that geometrical representation and relative positioning of sites are recorded to express the spatial organization arising in social and environmental data. A simple form of spatially referenced data is point data where observations are taken at fixed point sites of space and represented as triple $\left\{\left(x_{i}, y_{i}\right), z_{i}\right\}$, such that $\left(x_{i}, y_{i}\right)$ references the location of a point $i$ with respect to some coordinate system, while $z_{i}$ is the vector of measured attributes observed at site $i$. However, operations and activities of private and public institutions generally deal with space in terms of areas (irregular partitions or regular grid) and not points.

Areal data can be represented as point data by identifying each area with its centroid 24], but this is restrictive when observations for an area are descriptive of one or more (spatial) primary units, possibly of different type, collected 
within the same area boundary. In this case, data includes both attributes that relate to primary units or areas and attributes that refer to relations between primary units (e.g., contact frequencies between households) and between areal units (e.g., migration rates). Moreover, spatial-referencing poses a further degree of complexity due to the fact that the geometrical representation (point, line or polygon) and the relative positioning of primary units or areal units implicitly define spatial features (properties and relations) of different nature, that is, geometrical (e.g. area, distance), directional (e.g. north, south) and topological (e.g. crosses, on top) features. This relational information may be responsible for the spatial variation among areal units and it is extremely useful in descriptive modeling of different distributions holding for spatial subsets of data. An extra consequence is that observations across space cannot be considered independent due to the spatial continuity of events occurring in the space. Continuity of events over neighbor areas is a consequence of social patterns and environmental constraints that deal with space in terms of regions and allow to identify a mosaic of nearly homogeneous areas in which each patch of the mosaic is demarcated from its neighbors in terms of attributes levels. For instance, the spatial continuity of an environmental phenomenon such as air pollution may depend on the geographical arrangements of pollution sources. As a model for this spatial continuity, the regional concept encourages the analyst to exploit spatial correlation following from the first Law of Geography 22, according to which everything is related to everything else, but near things are more related than distant things. This means that primary units forming areal units of analysis will tend to be essentially identical members of same populations in nearby locations. In this spatial framework, relations among areal units of analysis are expressed in form of relational constraints that represent a discrete spatial structure arising in spatial data, while relations among primary units within an area model the spatial structure of each single areal unit of analysis.

Grouping connected areas to form clusters of homogeneous regions, i.e., spatial clustering, is a fundamental task of Spatial Data Mining. In this paper, we propose to represent the discrete spatial structure as a graph, where nodes are associated with relational descriptions of areal units to be clustered, while links express relational constraints which typically reflect spatial relations such as adjacency. In this way, discontinuity in the graph represents some obstacles in the space. Exploiting this graph-based representation, we present a clustering method, named CORSO (Clustering Of Related Structured Objects), that resorts to a multi-relational approach 2 to model homogeneity over relational structure embedded in spatial data and exploits the concept of graph neighborhood to capture relational constraints embedded in the graph edges. Units associated with (transitively) graph connected nodes are clustered together according to the similarity of their relational descriptions.

The paper is organized as follows. In the next section we discuss some related works. The method is presented in Section 3. Two applications of spatial clustering for topographic map interpretation and geo-referenced census data analysis are reported in Section 4, while conclusions are drawn in Section 5. 


\section{Background and Motivation}

The problem of clustering spatial data has been investigated by some researchers, but while a lot of research has been conducted on detecting spatial clusters from point data, only few works deal with areal data. For instance, $\mathrm{Ng}$ and Han [18] have proposed to extend the $k$-medoid partitioning algorithm [12] to group point data in a set of $k$ clusters. However, the $k$-medoid partitioning appears well suited only when spatial clusters are of convex shape and similar size, and the number $k$ is reasonably a-priori estimated. Moreover, the method suffers from severe limitations when clustering large spatial dataset 5 due to the complexity of computing distance between medoid points representing each pair of clusters. These efficiency drawbacks are partially alleviated when adopting both proximity and density information to achieve high quality spatial clusters in a sub-quadratic time without requiring the user to a-priori specify the number of clusters [7. Similarly, DBSCAN [6] exploits density information to efficiently detect clusters of arbitrary shape from point spatial data with noise. The key idea of density-based clustering is that for each point of a cluster, a neighborhood of a given radius has to contain a minimum number (cardinality) of data points. Neighborhood is determined according to the Euclidean distance. However, when observations concern areal units, Euclidean distance may not be appropriate to neighborhood determination. To this purpose, Sander et al. 21] have proposed GDBSCAN that generalizes DBSCAN in order to cluster not only point data but also spatially extended objects (lines or areas) taking into account both spatial and non spatial attributes when defining cardinality. Indeed, GDBSCAN extends the notion of neighborhood to any binary predicate that is symmetric and reflexive (e.g. distance, meet) and imposes a discrete spatial structure on data that guides the clustering detection. This discrete spatial structure can be equivalently modeled as links of a graph, namely neighborhood or proximity graph [23], whose nodes represent the units to be clustered. The graph-based representation of data, that is extensively used in pattern recognition [9, perfectly fits the spatial need of representing the relational constraints among spatial units to be clustered. In this perspective, it is clear that hybrid methods 17] which combine data clustering with graph-partitioning technique have some interesting applications properly in spatial clustering 8 .

However even when clustering takes into account relational constraints forming discrete spatial structure, all methods reported above suffer from severe limitations due to the single-table representation 2. Data to be clustered is represented in a single table (or relation) of a relational database, such that each row (or tuple) corresponds to a single unit of the sample population and the columns correspond to both spatial and a-spatial properties of these units. This representation is clearly inadequate when describing observations concerning several (spatial) primary units, eventually of different types, which are naturally modeled as many data tables as the number of object types and interactions. Some methods for mining clusters on (multi-)relational data have been investigated by resorting to the field of relational data mining. For instance, RDBC [13] forms clusters bottom-up in an agglomerative fashion that uses the distance metric 
introduced in [11] and handles relational representations with lists and other functional terms as well. In contrast, C0.5 1 adopts logical decision trees for clustering purposes by choosing split literals that maximize the distance between two resulting subsets (clusters) of examples. However, differently from RDBC, distance in literal choice is in this case estimated according to a user-provided propositional distance.

Although these relational clustering methods present several interesting aspects, detecting spatial clusters is a more complex task. Indeed, relational clustering methods generally work in the learning from interpretation setting [20] that allows to mine examples and background knowledge stored as Prolog programs exploiting expressiveness of first-order representation during clustering detection. The interpretation corresponding to each example $e$ given the background knowledge $B K$ is here intended as the minimal Herbrand model of $e \wedge B K$ and the implicit assumption is that separate interpretations are independent. This leads to ignore relational constraints eventually relating separate interpretations (e.g. geographic contiguity of areal units). This problem also occurs in graph-based relational learning methods [10] where graphs appear as a flexible representation for relational domains. However, these methods generally continue to work in learning from interpretation settings and thus ignore relations among graphs representing separate examples. In contrast, we propose to combine a graph-based partitioning algorithm with a relational clustering method to mine both relational constraints imposing the discrete spatial structure and relational data representing structured objects (spatial unit) to be clustered.

\section{The Method}

In a quite general formulation, the problem of clustering structured objects (e.g., complex areal units), which are related by links representing persistent relations between objects (e.g., spatial correlation), can be defined as follows: Given: (i) a set of structured objects $O$, (ii) a background knowledge $B K$ and (iii) a binary relation $R$ expressing links among objects in $O$; Find a set of homogeneous clusters $\mathbf{C} \subseteq \wp(O)$ that is feasible with $R$.

Each structured object $o_{i} \in O$ can be described by means of a conjunctive ground formula (conjunction of ground selectors) in a first-order formalism, while background knowledge $B K$ is expressed with first-order clauses that support some qualitative reasoning on $O$. In both cases, each basic component (i.e., selector) is a relational statement in the form $f\left(t_{1}, \ldots, t_{n}\right)=v$, where $f$ is a function symbol or descriptor, $t_{i}$ are terms (constant or variables) and $v$ is a value taken from the categorical or numerical range of $f$.

Structured objects are then related by $R$ that is a binary relation $R \subseteq O \times O$ imposing a discrete structure on $O$. In spatial domains, this relation may be either purely spatial, such as topological relations (e.g. adjacency of regions), distance relations (e.g. two regions are within a given distance), and directional relations (e.g. a region is on south of an other region), or hybrid, which mixes both spatial and non spatial properties (e.g. two regions are connected by a 
road). The relation $R$ can be described by the graph $G=\left(N_{O}, A_{R}\right)$ where $N_{O}$ is the set of nodes $n_{i}$ representing each structured object $o_{i}$ and $A_{R}$ is the set of $\operatorname{arcs} a_{i, j}$ describing links between each pair of nodes $\left\langle n_{i}, n_{j}\right\rangle$ according to the discrete structure imposed by $R$. This means that there is an arc from $n_{i}$ to $n_{j}$ only if $o_{i} R o_{j}$. Let $N_{R}\left(n_{i}\right)$ be the $R$-neighborhood of a node $n_{i}$ such that $N_{R}\left(n_{i}\right)=\left\{n_{j} \mid\right.$ there is an arc linking $n_{i}$ to $n_{j}$ in $\left.G\right\}$, a node $n_{j}$ is $R$-reachable from $n_{i}$ if $n_{j} \in N_{R}\left(n_{i}\right)$, or $\exists n_{h} \in N_{R}\left(n_{i}\right)$ such that $n_{j}$ is $R$-reachable from $n_{h}$.

According to this graph-based formalization, a clustering $\mathbf{C} \subseteq \wp(O)$ is feasible with the discrete structure imposed by $R$ when each cluster $C \in \mathbf{C}$ is a subgraph $G_{C}$ of the graph $G\left(N_{O}, A_{R}\right)$ such that for each pair of nodes $\left\langle n_{i}, n_{j}\right\rangle$ of $G_{C}, n_{i}$ is $R$-reachable from $n_{j}$, or vice-versa. Moreover, the cluster $C$ is homogeneous when it groups structured objects of $O$ sharing a similar relational description according to some similarity criterion.

CORSO integrates a neighborhood-based graph partitioning to obtain clusters which are feasible with $R$ discrete structure and resorts to a multi-relational approach to evaluate similarity among structured objects and form homogeneous clusters. This faces with the spatial issue of modeling spatial continuity of a phenomenon over the space. The top-level description of the method is presented in Algorithm 1. CORSO embeds a saturation step (function saturate) to make explicit information that is implicit in data according to the given BK. The key idea

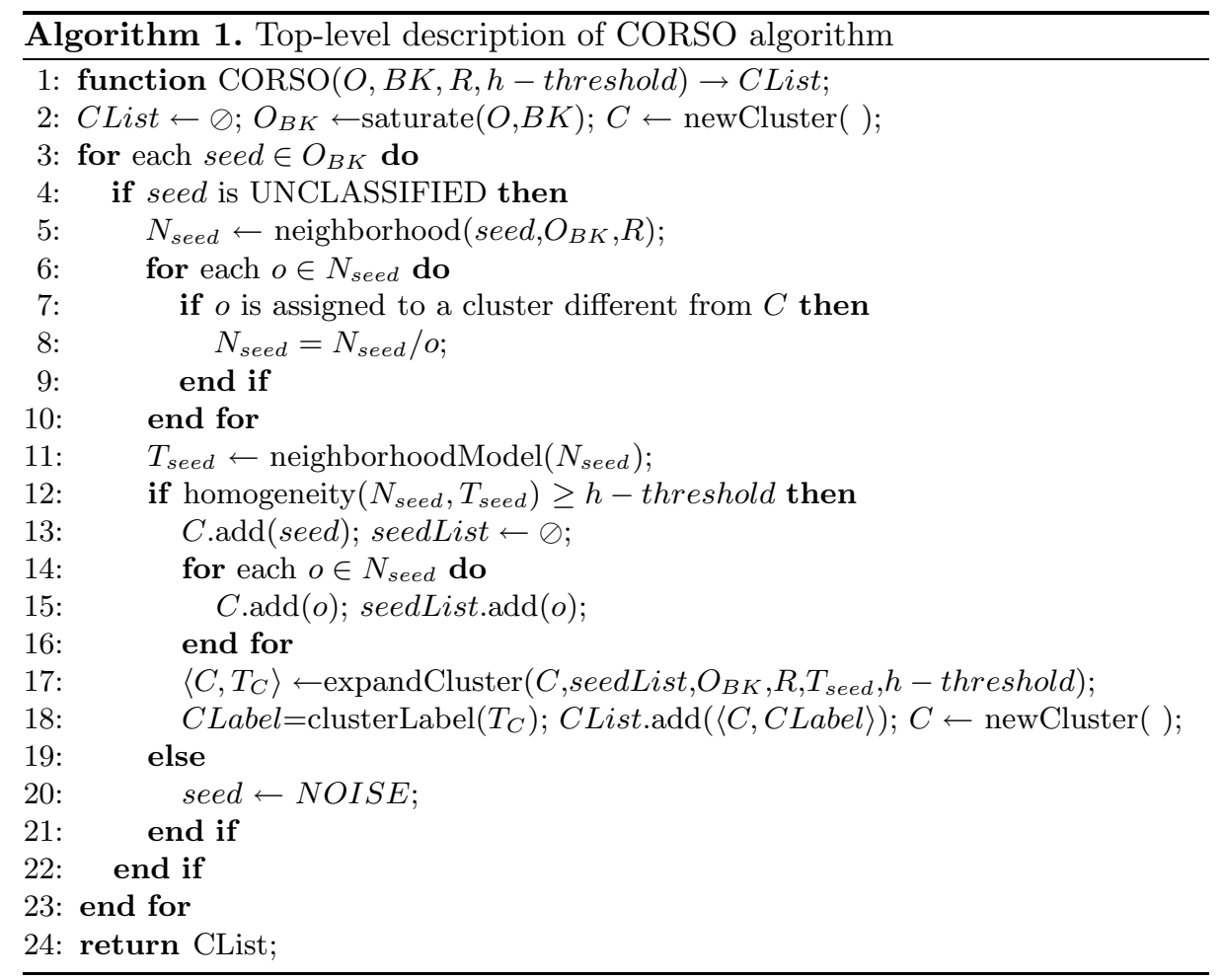


is to exploit the $R$-neighborhood construction and build clusters feasible with $R$ discrete structure by merging partially overlapping homogeneous neighborhood units. Cluster construction starts with an empty cluster $(C \leftarrow$ newCluster ()$)$ and chooses an arbitrary node seed from $G$. The $R$-neighborhood $N_{\text {seed }}$ of the node seed is then built according to $G$ discrete structure (function neighborhood) and the first-order theory $T_{\text {seed }}$ is associated to it. $T_{\text {seed }}$ is built as a generalization of the objects falling in $N_{\text {seed }}$ (function neighborhoodModel). When the neighborhood is estimated to be an homogeneous set (function homogeneity), cluster $C$ is grown with the structured objects enclosed in $N_{\text {seed }}$ whcih are not yet assigned to any cluster. The cluster $C$ is then iteratively expanded by merging the $R$-neighborhoods of each node of $C$ (neighborhood expansion) when these neighborhoods result in homogeneous sets with respect to current cluster model $T_{C}$ (see Algorithm 2.). $T_{C}$ is obtained as the set of first-order theories generalizing the neighborhoods merged in $C$. It is noteworthy that when a new $R$-neighborhood is built to be merged in $C$, all the objects which are already classified into a cluster different from $C$ are removed from the neighborhood. When the current cluster cannot be further expanded it is labeled with $C$ Label and an unclassified seed node for a new cluster is chosen from $G$ until all objects are classified. CLabel is obtained by $T_{C}$ (function labelCluster) to compactly describe $C$.

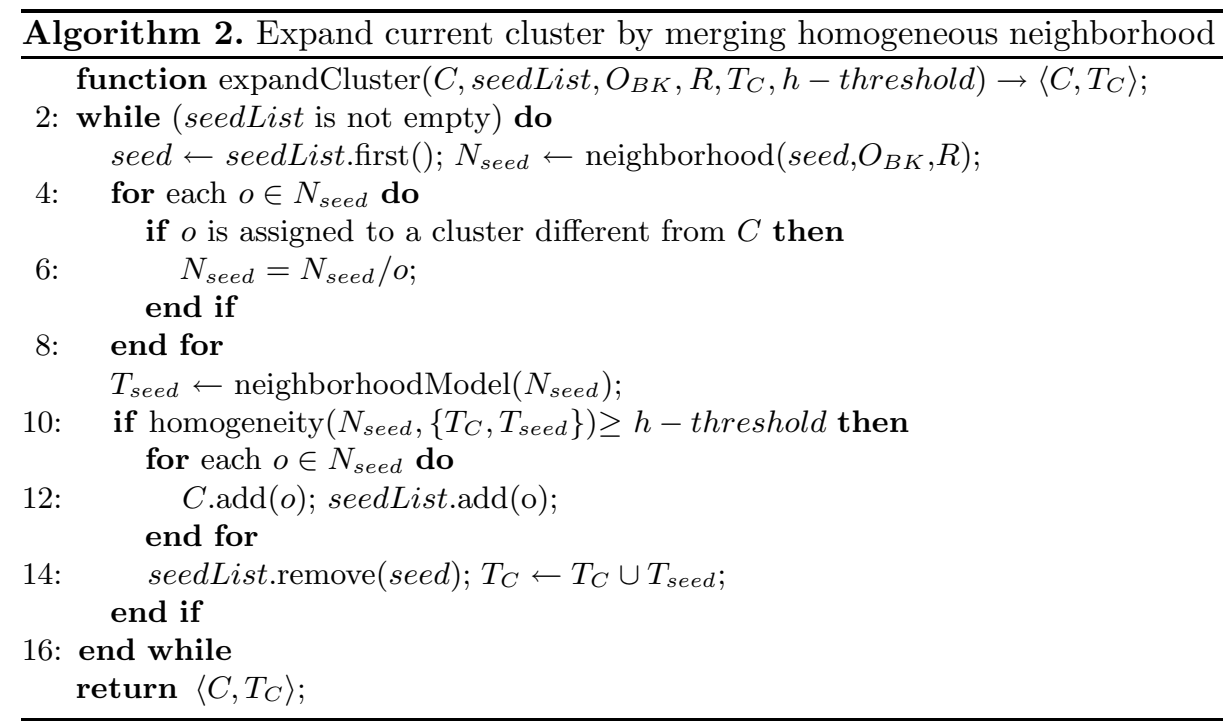

This is different from spatial clustering performed by GDBSCAN, although both methods share the neighborhood-based cluster construction. Indeed, GDBSCAN retrieves all objects density-reachable from an arbitrary core object by building successive neighborhoods and checks density within a neighborhood by ignoring the cluster. This yields a density-connected set, where density is 
efficiently estimated independently from the neighborhoods already merged in forming the current cluster. However, this approach may lead to merge connected neighborhoods sharing some objects but modeling different phenomena. Moreover, GDBSCAN computes density within each neighborhood according to a weighted cardinality function (e.g. aggregation of non spatial values) that assumes single table data representation. CORSO overcomes these limitations by computing density within a neighborhood in terms of degree of similarity among all relationally structured objects falling in the neighborhood with respect to the model of the entire cluster currently built. In particular, following the suggestion given in [16, we evaluate homogeneity within a neighborhood $N_{\text {seed }}$ to be added to the cluster $C$ as the average degree of matching between objects of $N_{\text {seed }}$ and the cluster model $\left\{T_{C}, T_{\text {seed }}\right\}$. Details on cluster model determination, neighborhood homogeneity estimation and cluster labeling are reported below.

\subsection{Cluster Model Generation}

Let $C$ be the cluster currently built by merging $w$ neighborhood sets $N_{1}, \ldots, N_{w}$, we assume that the cluster model $T_{C}$ is a set of first-order theories $\left\{T_{1}, \ldots, T_{w}\right\}$ for the concept $C$ where $T_{i}$ is a model for the neighborhood set $N_{i}$. More precisely, $T_{i}$ is a set of first-order clauses: $T_{i}:\left\{\operatorname{cluster}(X)=c \leftarrow H_{i 1}, \ldots, \operatorname{cluster}(X)=\right.$ $c \leftarrow H_{i z}$, where each $H_{i j}$ is a conjunctive formula describing a sub-structure shared by one or more objects in $N_{i}$ and $\forall o_{i} \in N_{i}, B K \cup T_{i} \models o_{i}$. Such model can be learned by resorting to the ILP system ATRE 14 that adopts a separate-andconquer search strategy to learn a model of structured objects from a set of training examples and eventually counter-examples. In this context, ATRE learns a model for each neighborhood set without considering any counter-examples. The search of a model starts with the most general clause, that is, cluster $(X)=c \leftarrow$, and proceeds top-down by adding selectors (literals) to the body according to some preference criteria (e.g. number of objects covered or number of literals).

Selectors involving both numerical and categorical descriptors are handled in the same way, that is, they have to comply with the property of linkedness and are sorted according to preference criteria. The only difference is that selectors involving numerical descriptors are generalized by computing the closed interval that best covers positive examples and eventually discriminates from contourexamples, while selectors involving categorical descriptors with same function value are generalized by simply turning all ground arguments into corresponding variables without changing the corresponding function value.

\subsection{Neighborhood Homogeneity Estimation}

The homogeneity of a neighborhood set $N$ to be added to the cluster $C$ is computed as follows:

$$
h\left(N, T_{C \cup N}\right)=\frac{1}{\# N} \sum_{i} h\left(o_{i}, T_{C \cup N}\right)=\frac{1}{\# N} \sum_{i} \frac{1}{w+1} \sum_{j} h\left(o_{i}, T_{j}\right),
$$


where $\# N$ is the cardinality of the neighborhood set $N$ and $T_{C \cup N}$ is the cluster model of $C \cup N$ formed by both $\left\{T_{1}, \ldots, T_{w}\right\}$, i.e., the model of $C$ and $T_{w+1}$, i.e., the model of $N$ built as explained above. Since $T_{j}=H_{1 j}, \ldots, H_{z j}(z \geq 1)$ with each $H_{i j}$ a conjunctive formula in first-order formalism, we assume that:

$$
h\left(o_{i}, T_{j}\right)=\frac{1}{z} \sum_{i} f m\left(o_{i}, H_{i j}\right),
$$

where $f m$ is a function returning the degree of matching of an object $o_{i} \in N$ against the conjunctive formula $H_{i j}$. In this way, the definition of homogeneity of a neighborhood set $N=\left\{o_{1}, \ldots, o_{n}\right\}$ with respect to some logical theory $T_{C \cup N}$ is closely related to the problem of comparing (matching) the conjunctive formula $f_{i}$ representing an object $o_{i} \in N$ 1 with a conjunctive formula $H_{i j}$ forming the model $T_{j}$ in order to discover likenesses or differences [19]. This is a directional similarity judgment involving a referent $R$, that is the description or prototype of a class (cluster model) and a subject $S$ that is the description of an instance of a class (object to be clustered). In the classical matching paradigm, the matching of $S$ against $R$ corresponds to compare them just for equality. In particular, when both $S$ and $R$ are conjunctive formulas in first-order formalism, matching $S$ against $R$ corresponds to check the existence of a substitution $\theta$ for the variables in $R$ such that $S=\theta(R)$. This last condition is generally weakened by requiring that $S \Rightarrow \theta(R)$, where $\Rightarrow$ is the logical implication. However, the requirement of equality, even in terms of logical implication, is restrictive in presence of noise or variability of the phenomenon described by the referent of matching. This makes necessary to rely on a flexible definition of matching that aims at comparing two descriptions and identifying their similarities rather than equalities. The result of such a flexible matching is a number in the interval $[0,1]$ that is the probability of precisely matching $S$ against $R$, provided that some change described by $\theta$ is possibly made in the description $R$.

The problem of computing flexible matching to compare structures is not novel. Esposito et al. 4] have formalized a computation schema for flexible matching on formulas in first-order formalism whose basic components (selectors) are the relational statements, that is, $f_{i}\left(t_{1}, \ldots, t_{n}\right)=v$, which are combined by applying different operators such as conjunction $(\wedge)$ or disjunction $(\vee)$ operator. In this work, we focus on the computation of flexible matching $f m(S, R)$ when both $S$ and $R$ are described by conjunctive formulas and $\operatorname{fm}(S, R)$ looks for the substitution $\theta$ returning the best matching of $S$ against $R$, as:

$$
f m(S, R)=\max _{\theta} \prod_{i=1, \ldots, k} f m_{\theta}\left(S, r_{i}\right) .
$$

The optimal $\theta$ that maximizes the above conditional probability is here searched by adopting the branch and bound algorithm that expands the least cost partial path by performing quickly on average [4. According to this formulation, $f m_{\theta}$

\footnotetext{
${ }^{1}$ The conjunctive formula $f_{i}$ is here intended as the description of $o_{i} \in N$ saturated according to the $B K$.
} 
denotes the flexible matching with the tie of the substitution fixed by $\theta$ computed on each single selector $r_{i} \equiv f_{r_{i}}\left(t_{r_{1}}, \ldots, t_{r_{n}}\right)=v_{r_{i}}$ of the referent $R$ where $f_{r_{i}}$ is a function descriptor with either numerical (e.g. area or distance) or categorical (e.g. intersect) range. In the former case the function value $v_{r_{i}}$ is an interval value $\left(v_{r_{i}} \equiv[a, b]\right)$, while in the latter case $v_{r_{i}}$ is a subset of values $\left(v_{r_{i}} \equiv\right.$ $\left.\left\{v_{1}, \ldots, v_{M}\right\}\right)$ from the range of $f_{r_{i}}$. This faces with a referent $R$ that is obtained by generalizing a neighborhood of objects in $O$. Conversely for the subject $S$, that is, the description of a single object $o \in O$, the function value $w_{s_{j}}$ assigned to each selector $s_{j} \equiv f_{s_{j}}\left(t_{s_{1}}, \ldots, t_{s_{n}}\right)=w_{s_{j}}$ is an exactly known single value from the range of $f_{s_{j}}$. In this context, the flexible matching $f m_{\theta}\left(S, r_{i}\right)$ evaluates the degree of similarity $f m\left(s_{j}, \theta\left(r_{i}\right)\right)$ between $\theta\left(r_{i}\right)$ and the corresponding selector $s_{j}$ in the subject $S$ such that both $r_{i}$ and $s_{j}$ have the same function descriptor $f_{r}=f_{s}$ and for each pair of terms $\left\langle t_{r_{i}}, t_{s_{i}}\right\rangle, \theta\left(t_{r_{i}}\right)=t_{s_{i}}$. More precisely,

$$
f m\left(s_{j}, \theta\left(r_{i}\right)\right)=f m\left(w_{s_{j}}, v_{r_{i}}\right)=\max _{v \in v_{r_{i}}} P\left(\operatorname{equal}\left(w_{s_{j}}, v\right)\right) .
$$

The probability of the event equal $\left(w_{s_{j}}, v\right)$ is then defined as the probability that an observed $w_{s_{j}}$ is a distortion of $v$, that is:

$$
P\left(\operatorname{equal}\left(w_{s_{j}}, v\right)\right)=P\left(\delta(X, v) \geq \delta\left(w_{s_{j}}, v\right)\right)
$$

where $X$ is a random variable assuming value in the domain $D$ representing the range of $f_{r}$ while delta is a distance measure. The computation of $P\left(\operatorname{equal}\left(w_{s_{j}}, v\right)\right)$ clearly depends on the probability density function of $X$. For categorical descriptors, that is, $D$ is a discrete set with cardinality $\# D$, it has be proved [4] that:

$$
P(\operatorname{equal}(w, v))=\left\{\begin{array}{l}
1 \text { if } w_{s_{j}}=v \\
\# D-1 / \# D \text { otherwise }
\end{array}\right.
$$

when $X$ is assumed to have a uniform probability distribution on $D$ and $\delta(x, y)=$ 0 if $x=y, 1$ otherwise. Although similar results have been reported for both linear non numerical and tree-structured domains, no result appears for numerical domains. Therefore, we have extended definitions reported in 4 to make flexible matching able to deal with numerical descriptors and we have proved that:

$$
f m(c,[a, b])= \begin{cases}1 & \text { if } a \leq c \leq b \\ 1-2(a-c) /(\beta-\alpha) & \text { if } c<a \wedge 2 a-c \leq \beta \\ (c-\alpha) /(\beta-\alpha) & \text { if } c<a \wedge 2 a-c>\beta \\ (\beta-c) /(\beta-\alpha) & \text { if } c>b \wedge 2 b-c<\alpha \\ 1-2(c-b) /(\beta-\alpha) & \text { if } c>b \wedge 2 b-c \geq \alpha\end{cases}
$$

by assuming that $X$ has uniform distribution on $D$ and $\delta(x, y)=|x-y|$. A proof of formula 7 is reported in the Appendix $\mathrm{A}$ of this paper.

\subsection{Cluster Labeling}

A cluster $C$ can be naturally labeled with $T_{C}$ that is the set of first-order clauses obtained from the generalization of neighborhoods merged in $C$. Each first-order 
clause is in the form $C \leftarrow s_{1}, \ldots, s_{n}$, where $C$ represents the cluster label and each $s_{i}$ denotes a selector in the form $f_{i}\left(t_{i_{1}}, \ldots, t_{i_{l}}\right)=v_{i}$. In this formalization, two selectors $s_{1}: f_{1}\left(t_{1_{1}}, \ldots, t_{1_{l}}\right)=v_{1}$ and $s_{2}: f_{2}\left(t_{2_{1}}, \ldots, t_{2_{l}}\right)=v_{2}$ are comparable according to some substitution $\theta$ when they involve the same descriptor $\left(f_{1}=f_{2}=f\right)$ and each pair of terms $\left\langle t_{1_{i}}, t_{2_{i}}\right\rangle$ is unifiable according to $\theta$, i.e., $t_{1_{i}} \theta=t_{2_{i}} \theta=t_{i}(\forall i=1 \ldots l)$. In this case, the selector $s: f\left(t_{1}, \ldots, t_{l}\right)=\left\{v_{1}\right\} \cup\left\{v_{2}\right\}$ is intended as a generalization for both $s_{1}$ and $s_{2}$. In particular, the selectors $s_{1}$ and $s_{2}$ are equal when they are comparable and $v_{1}=v_{2}=v$ such that the generalization of $s_{1}$ and $s_{2}$ is built as $s: f\left(t_{1}, \ldots, t_{l}\right)=v$. Similarly, the selector $s_{1}\left(s_{2}\right)$ is contained in the selector $s_{2}\left(s_{1}\right)$ when they are comparable and $v_{1} \subseteq v_{2}\left(v_{2} \subseteq v_{1}\right)$, while the generalization $s$ is $f\left(t_{1}, \ldots, t_{l}\right)=v_{2}\left(f\left(t_{1}, \ldots, t_{l}\right)=v_{1}\right)$. Note that equality of selectors implies containment, but not vice-versa. Similarly, the first-order clauses $H_{1}: C \leftarrow s_{1_{1}}, \ldots, s_{1_{n}}$ and $H_{2}: C \leftarrow s_{2_{1}}, \ldots, s_{2_{n}}$ are comparable according to some substitution $\theta$ when each pair of selectors $\left\langle s_{1_{i}}, s_{2_{i}}\right\rangle$ is comparable according to $\theta$. Hence, $H_{1}$ is equal (contained) to $H_{2}$ when $s_{1_{i}}$ is equal (contained) to $s_{2_{i}}$ for each $i=1, \ldots, n$. In both these cases (equality and containment condition), the pair of first-order clauses $H_{1}, H_{2}$ can be replaced without lost of information with the first-order clause $H$ that is the generalization of $H_{1}, H_{2}$ built by substituting each pair of comparable selectors $\left\langle s_{1_{i}}, s_{2_{i}}\right\rangle \in\left\langle H_{1}, H_{2}\right\rangle$ with the generalization obtained as stated above. This suggests the idea of merging a pair of comparable first-order clauses $H_{1}, H_{2}$ in a single clause $H$ by preserving the equivalence of coverage, that is: (i) for each structured object $o$ with $H_{1}, H_{2}, B K \models o$ then $H, B K \models o$ and vice-versa, (ii) for each structured object $o$ with $H_{1}, H_{2}, B K \not \models o$ then $H, B K \not \models o$ and vice-versa, where $B K$ is a set of first-order clauses. The equivalence of coverage between $\left\{H_{1}, H_{2}\right\}$ and $H$ is obviously guaranteed when $H_{1}$ is either equal or contained in $H_{2}$ or vice-versa, but this equivalence cannot be guaranteed when $H_{1}$ and $H_{2}$ are comparable first-order clauses but neither equality condition nor containment condition are satisfied.

Example 1: Let us consider the pair of comparable first-order clauses:

$$
\begin{aligned}
& H_{1}: \operatorname{cluster}\left(X_{1}\right)=c \leftarrow \text { distance }\left(X_{1}, X_{2}\right)=[5 . .10] \text {, type }\left(X_{2}\right)=\text { street } \\
& H_{2}: \operatorname{cluster}\left(X_{1}\right)=c \leftarrow \operatorname{distance}\left(X_{1}, X_{2}\right)=[3 . .7] \text {,type }\left(X_{2}\right)=\text { river }
\end{aligned}
$$

where neither $H_{1}$ is equal to $H_{2}$ nor $H_{1}\left(H_{2}\right)$ is contained in $H_{2}\left(H_{1}\right)$. The firstorder clause obtained by generalizing pairs of comparable selectors in both $H_{1}$ and $H_{2}$, is $H: \operatorname{cluster}\left(X_{1}\right)=c \leftarrow \operatorname{distance}\left(X_{1}, X_{2}\right)=[3 . .10]$, type $\left(X_{2}\right)=$ $\{$ street, river $\}$, where $H \models o$ with $o$ : distance $\left(X_{1}, X_{2}\right)=3 \wedge$ type $\left(X_{2}\right)=$ street, but neither $H_{1} \models o$ nor $H_{2} \models o$.

The requirement of equality between $H_{1}$ and $H_{2}$ can be relaxed while preserving equivalence of coverage with respect to the generalization $H$. Indeed, when

$$
\begin{aligned}
& H_{1}: C \leftarrow s_{1}(-)=v_{1}, \ldots s_{k}(-)=v_{k}, \ldots, s_{n}(-)=v_{n} \\
& H_{2}: C \leftarrow s_{1}(-)=v_{1}, \ldots s_{k}(-)=w_{k}, \ldots, s_{n}(-)=v_{n}
\end{aligned}
$$

are comparable first-order clauses differing only in the function value of a single selector (i.e. $s_{k}$ ), the first-order clause: 
$H: C \leftarrow s_{1}\left({ }_{-}\right)=v_{1}, \ldots s_{k}\left({ }_{-}\right)=\left\{v_{k}\right\} \cup\left\{w_{k}\right\}, \ldots, s_{n}\left({ }_{-}\right)=v_{n}$ continues to preserve the equivalence of coverage with $\left\{H_{1}, H_{2}\right\}$.

Example 2: Let us consider the pair of comparable first-order clauses:

$$
\begin{aligned}
H_{1}: \text { cluster }\left(X_{1}\right)= & c \leftarrow \operatorname{distance}\left(X_{1}, X_{2}\right)=[3 . .7], \text { type }\left(X_{2}\right)=\text { street }, \\
& \text { length }\left(X_{2}\right)=[3,5] \\
H_{2}: \operatorname{cluster}\left(X_{1}\right)= & c \leftarrow \operatorname{distance}\left(X_{1}, X_{2}\right)=[3 . .7], \operatorname{type}\left(X_{2}\right)=\text { street }, \\
& \text { length }\left(X_{2}\right)=[7,10]
\end{aligned}
$$

which differ only in the value of a single selector (length), the first-order clause obtained by generalizing the pairs of comparable selectors in both $H_{1}$ and $H_{2}$ is:

$$
\begin{gathered}
H: \text { cluster }\left(X_{1}\right)=c \leftarrow \text { distance }\left(X_{1}, X_{2}\right)=[3 . .7], \text { type }\left(X_{2}\right)=\text { street }, \\
\text { length }\left(X_{2}\right)=[3,5] \cup[7,10]
\end{gathered}
$$

that is equivalent in coverage to the pair $\left\{H_{1}, H_{2}\right\}$.

Following this idea, it is possible to compactly describe the cluster theory $T_{C}$ finally associated to a cluster $C$ by iteratively replacing pairs of comparable first-order clauses $H_{1}, H_{2}$ with the generalization $H$, when $H$ results equivalent in coverage to $\left\{H_{1}, H_{2}\right\}$ (see Algorithm 3.).

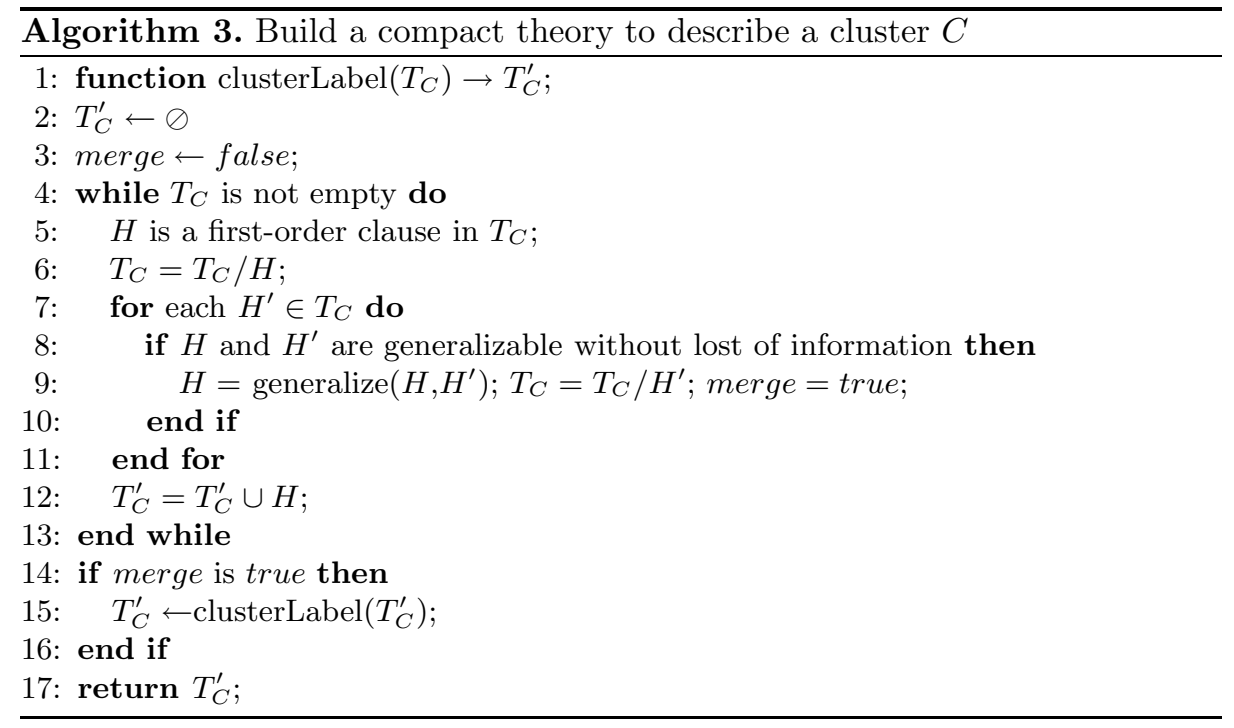

Example 3: Let us consider $T_{C}$ that is the set of first-order clauses including:

$H_{1}: \operatorname{cluster}\left(X_{1}\right)=c \leftarrow$ distance $\left(X_{1}, X_{2}\right)=[5 . .10]$, color $\left(X_{2}\right)=$ red

$H_{2}: \operatorname{cluster}\left(X_{1}\right)=c \leftarrow$ distance $\left(X_{1}, X_{2}\right)=[5 . .6], \operatorname{color}\left(X_{2}\right)=$ blue

$H_{3}: \operatorname{cluster}\left(X_{1}\right)=c \leftarrow$ distance $\left(X_{1}, X_{2}\right)=[5 . .10], \operatorname{color}\left(X_{2}\right)=$ blue

$H_{4}: \operatorname{cluster}\left(X_{1}\right)=c \leftarrow \operatorname{distance}\left(X_{1}, X_{2}\right)=[6 . .10]$, area $\left(X_{2}\right) \operatorname{in}[30 . .40]$

$T_{C}$ can be transformed in the set of first-order clauses:

$$
\begin{aligned}
& H_{1}^{\prime}: \text { cluster }\left(X_{1}\right)=c \leftarrow \text { distance }\left(X_{1}, X_{2}\right)=[5 . .10], \text { color }\left(X_{2}\right)=\{\text { red, blue }\} \\
& H_{2}^{\prime}: \operatorname{cluster}\left(X_{1}\right)=c \leftarrow \text { distance }\left(X_{1}, X_{2}\right)=[6 . .10], \text { area }\left(X_{2}\right) \text { in }[30 . .40]
\end{aligned}
$$


where $H_{1}^{\prime}$ results by firstly merging $H_{1}$ and $H_{3}$, which are comparable and differ only in the function value of a selector $\left(\operatorname{color}\left(X_{2}\right)=\right.$ red vs color $\left(X_{2}\right)=$ blue $)$, and obtaining $H_{13}:$ cluster $\left(X_{1}\right)=c \leftarrow \operatorname{distance}\left(X_{1}, X_{2}\right)=[5 . .10], \operatorname{color}\left(X_{2}\right)=$ \{red,blue $\}$ and then merging $H_{13}$ and $H_{2}$ since $H_{2}$ is contained in $H_{13}$.

\section{The Application: Two Case Studies}

In this section, we describe the application of CORSO to two distinct real-world problems, namely topographic map interpretation and geo-referenced census data analysis. In the former problem, a topographic map is treated as a grid of square cells of same size, according to a hybrid tessellation-topological model such that adjacency among cells allows map-reading from a cell to one of its neighbors in the map. For each cell, geographical data is represented as humans perceive it in reality, that is, geometric (or physical) representation and thematic (or logical) representation. Geometric representation describes the geographical objects by means of the most appropriate physical entity (point, line or region), while thematic representation expresses the semantics of geographical objects (e.g., hydrography, vegetation, transportation network and so on), independently of their physical representation. Spatial clustering aims at identifying a mosaic of nearly homogeneous clusters (areas) including adjacent cells in the map such that geographical data inside each cluster properly models the spatial continuity of some morphological environment within the cluster region, while separate clusters model spatial variation over the entire space. In the second problem, the goal is to perform a joint analysis of both socio-economic factors represented in census data and geographical factors represented in topographic maps to support a good public policy. In this case, spatial objects are territorial units for which census data are collected as well as entities of geographical layers such as urban and wood areas. Spatial partitioning of CORSO is compared with the first-order clustering performed with logical decision trees [1, which are able to manage relational structure of spatial objects but ignore relations imposed with discrete spatial structure. The empirical comparison with GDBSCAN was not possible since the system is not publicly available. However, CORSO clearly improves GDBSAN clustering that is not able to manage complex structure of spatial data. In both applications, running time of CORSO refers to execution performed on a $2 \mathrm{Ghz}$ IBM notebook with $256 \mathrm{Mb}$ of RAM.

\subsection{Topographic Map Interpretation}

In this study we discuss two real-world applications of spatial clustering to characterize spatial continuity of some morphological elements over the topographic map of the Apulia region in Italy. The territory considered in this application covers $45 \mathrm{~km}^{2}$ from the zone of Canosa in Apulia. The examined area is segmented into square areal units of $1 \mathrm{Km}^{2}$ each. Thus, the problem of recognizing spatial continuity of some morphological elements in the map is reformulated as the problem of grouping adjacent cells resulting in a morphologically homogeneous area, that is, a problem of clustering spatial objects according to the 

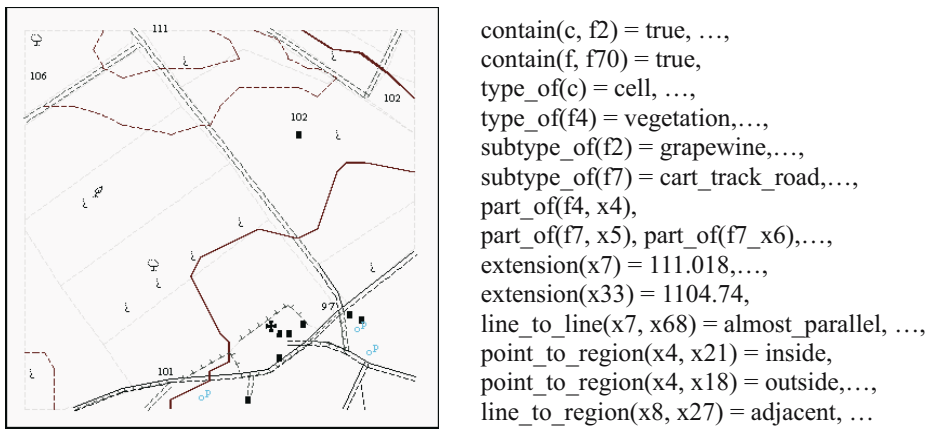

Fig. 1. First-order description of a cell extracted from topographic chart of Apulia

discrete spatial structure imposed by the relation of "adjacency" among cells. Since several geographical objects, eventually belonging to different layers (e.g., almond tree, olive tree, font, street, etc) are collected within each cell, we apply algorithms derived from geometrical and topological reasoning [15] to obtain cell descriptions in first-order formalism (see Figure 11). For this task, we consider descriptions including spatial descriptors encompassing geometrical properties (area, extension) and topological relations (regionToRegion, lineToLine, pointToRegion) as well as non spatial descriptors (typeOf, subtypeOf). The descriptor part $O f$ is used to define the physical structure of a logical object. An example is: typeOf $\left(f_{1}\right)=$ font $\wedge$ partO $f\left(f_{1}, x_{1}\right)=$ true, where $f_{1}$ denotes a font which is physically represented by a point referred with the constant $x_{1}$. Each cell is here described by a conjunction of 946,866 ground selectors in average. To support some qualitative reasoning, a spatial background knowledge (BK) is expressed in form of clauses. An example of BK we use in this task is:

fontToParcel(Font, Culture $)=$ Relation $\leftarrow$ typeOf $($ Font $)=$ font , partOf $($ Font, Point $)=$ true, typeOf $($ Parcel $)=$ parcel ,

partOf $($ Parcel, Region $)=$ true, pointToRegion $($ Point, Region $)=$ Relation that allows to move from a physical to a logical level in describing the topological relation between the point that physically represents the font and the region that physically represents the culture and that are, respectively, referred to as the variables Font and Culture. The specific goal of this study is to model the spatial continuity of some morphological environment (e.g. cultivation setting) within adjacent cells over the map. This means that each cluster covers a contiguous area over the map where it is possible to observe some specific environment that does not occur in adjacent cells not yet assigned to any cluster. It is noteworthy that granularity of partitioning changes by varying homogeneity threshold (see Figure 2). In particular, when $h-$ threshold $=0.95$, CORSO clusters adjacent cells in five regions in 1821 secs. Each cluster is compactly labeled as follows: $C_{1}$ : cluster $\left(X_{1}\right)=c_{1} \leftarrow$ containAlmondTree $\left(X_{1}, X_{2}\right)=\{$ true $\}$, cultivationToCulture $\left(X_{2}, X_{3}\right)=\{$ outside $\}$, areaCulture $\left(X_{3}\right)=[328 . .420112]$, fontToCulture $\left(X_{4}, X_{3}\right)=\{$ outside $\}$. 

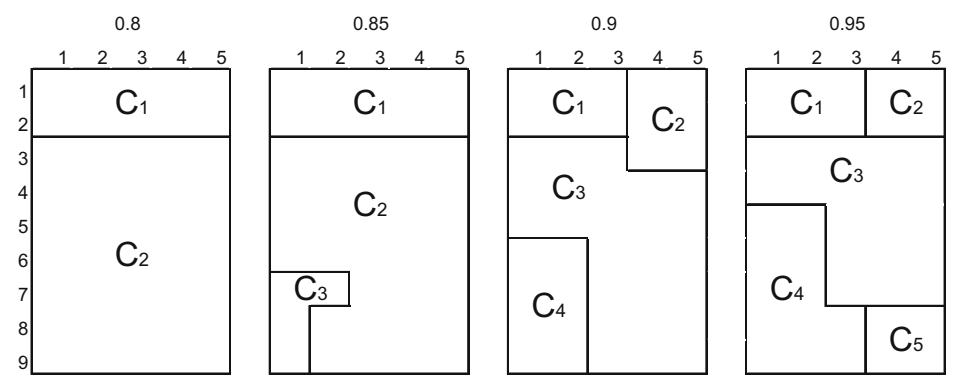

Fig. 2. Spatial clusters detected on map data from the zone of Canosa by varying $h-$ threshold value in $\{0.8,0.85,0.9,0.95\}$

$$
\begin{aligned}
& C_{2}: \text { cluster }\left(X_{1}\right)=c_{2} \leftarrow \text { containAlmondTree }\left(X_{1}, X_{2}\right)=\{\text { true }\}, \\
& \text { cultivationToCulture }\left(X_{2}, X_{3}\right)=\{\text { inside }\} \text {, areaCulture }\left(X_{3}\right)=[13550 . . \\
& \text { 187525], areaCulture }\left(X_{3}\right)=[13550.187525], \\
& \text { cultivationToCulture }\left(X_{2}, X_{4}\right) \in\{\text { outside }\} . \\
& C_{3}: \text { cluster }\left(X_{1}\right)=c_{3} \leftarrow \text { containGrapevine }\left(X_{1}, X_{2}\right)=\{\text { true }\}, \\
& \text { cultivationToCulture }\left(X_{2}, X_{3}\right)=\{\text { inside }\}, \text { areaCulture }\left(X_{3}\right)=[13550 . . \\
& 212675], \text { cultivationToCulture }\left(X_{2}, X_{4}\right)=\{\text { outside }\} . \\
& \text { cluster }\left(X_{1}\right)=c_{3} \leftarrow \text { containGrapevine }\left(X_{1}, X_{2}\right)=\{\text { true }\}, \\
& \text { cultivationToCulture }\left(X_{2}, X_{3}\right)=\{\text { outside }\} \text {, areaCulture }\left(X_{3}\right)=[150 . . \\
& 212675], \text { cultivationToCulture }\left(X_{2}, X_{4}\right)=\{\text { outside, inside }\} . \\
& C_{4}: \text { cluster }\left(X_{1}\right)=c_{4} \leftarrow \text { containStreet }\left(X_{1}, X_{2}\right)=\{\text { true }\} \\
& \text { streetToCulture }\left(X_{2}, X_{3}\right)=\{\text { adjacent }\}, \text { areaCulture }\left(X_{3}\right)=[620 . . \\
& \text { 230326], cultureToCulture }\left(X_{3}, X_{4}\right)=\{\text { outside, inside }\} . \\
& C_{5}: \text { cluster }\left(X_{1}\right)=c_{5} \leftarrow \text { containOliveTree }\left(X_{1}, X_{2}\right)=\text { true, } \\
& \text { cultivationToCulture }\left(X_{2}, X_{3}\right) \in\{\text { outside }\}, \text { areaCulture }\left(X_{3}\right) \in[620 . . \\
& \text { 144787 }], \text { oliviToParcel }\left(X_{2}, X_{4}\right)=\{\text { outside }\} .
\end{aligned}
$$

Notice that each detected cluster effectively includes adjacent cells sharing a similar morphological environment, while separate clusters describe quite different environments. Conversely, the logical decision tree mined on the same data divides the territory under analysis in twenty different partitions where it is difficult to recognize the continuity of any morphology phenomenon.

\subsection{Geo-referenced Census Data Analysis}

In this application, we consider both census and digital map data concerning North West England (NWE) area that is decomposed into censual sections or wards for a total of 1011 wards. Census data is available at ward level and provides some measures of deprivation level in the ward according to index scores that combine information provided by 1998 Census. We consider Jarman Underprivileged Area Score that is designed to measure the need for primary care, the indices developed by Townsend and Carstairs that is used in health-related analysis, and the Department of the Environment's Index (DoE) that is used 
in targeting urban regeneration funds. The higher the index value the more deprived a ward is. Spatial analysis of deprivation distribution is enabled by the availability of vectorized boundaries of the 1010 census wards as well as by other Ordnance Survey digital maps of NWE, where several interesting layers are found, namely urban zones (including 384 large urban areas and 2232 small urban areas) and wood zones (including 859 woods). In particular, we focus attention on investigating continuity of socio-economic deprivation joined to geographical factors represented in linked topographic maps.

Both ward-referenced census data and map data are stored in an ObjectRelational spatial database, i.e., Oracle Spatial 9i database, as a set of spatial tables, one for each layer. Each spatial table includes a geometry attribute that allows storing the geometrical representation (i.e. urban and wood zones are described by lines while wards are described by polygons) and the positioning of a spatial object with respect to some reference system. We adopt a topological algorithm based on the 9-intersection model 3 to detect both adjacency relation between NWE wards (i.e. wards which share some boundary) and overlapping relation between wards and urban areas (or woods). The former imposes a discrete spatial structure over NWE wards such that only adjacent wards may be grouped in the same cluster while the latter contributes to define the spatial structure embedded in each ward not only in terms of observed values of deprivation scores but also extension of urban areas and/or woods overlapping each ward. No $B K$ is defined for this problem.

Granularity of partitioning changes when varying the value of $h$-threshold, that is, CORSO detects 79 clusters with $h$-threshold $=0.80,89$ clusters with $h-$ threshold $=0.85,122$ clusters with $h-$ threshold $=0.90$ and 163 clusters with $h$-threshold $=0.95$. In particular, when $h-$ threshold $=0.95$, CORSO clusters NWE area in 2160 secs and identifies adjacent regions modeling differently relational patterns involving deprivation and geographical environment. For instance, by analyzing these spatial clusters, we discover three adjacent areas, namely $C_{1}, C_{2}$ and $C_{3}$ compactly labeled as follows:

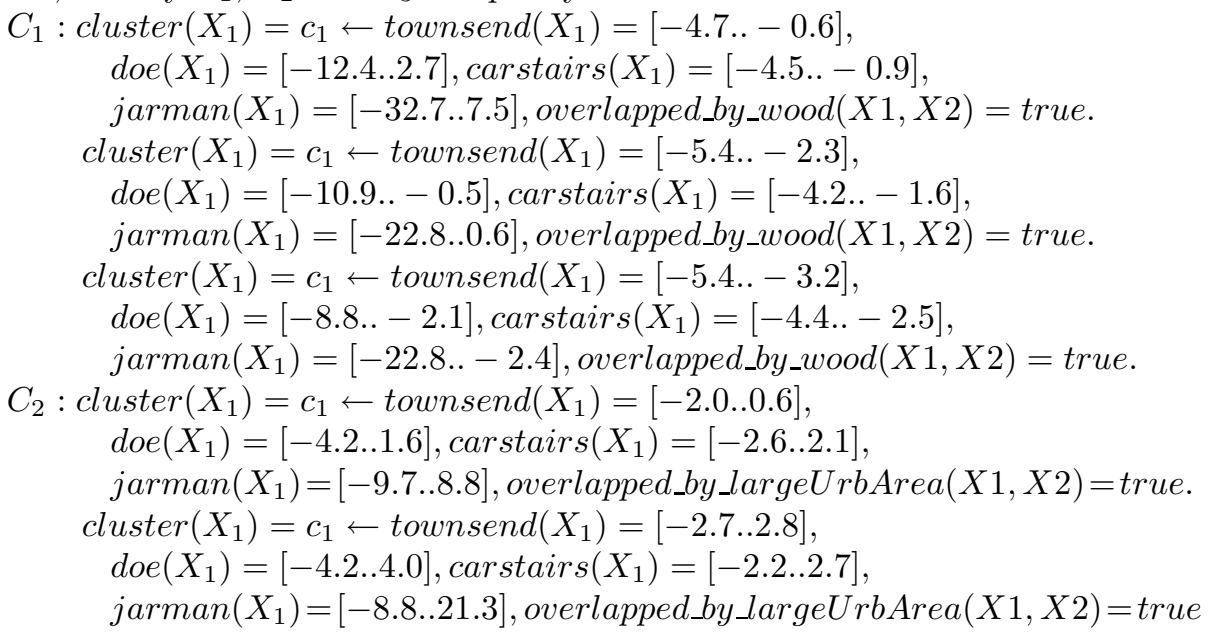




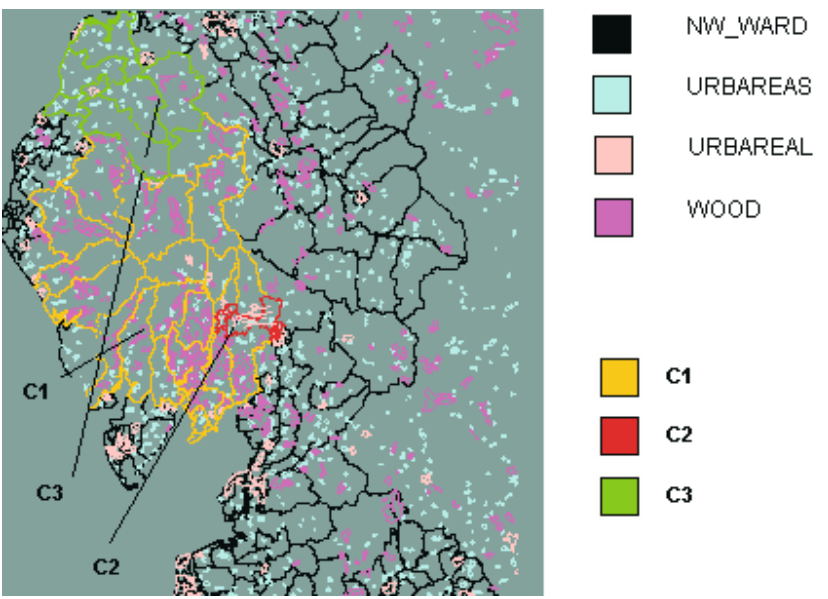

Fig. 3. Spatial clusters detected on NWE with $h-$ threshold $=0.95$

$$
\begin{aligned}
& C_{3}: \text { cluster }\left(X_{1}\right)=c_{1} \leftarrow \text { townsend }\left(X_{1}\right)=[-3.4 . .0 .4], \\
& \quad \text { doe }\left(X_{1}\right)=[-8.2 . .-0.2], \text { carstairs }\left(X_{1}\right)=[-3.7 . .0 .6], \\
& \quad \text { jarman }\left(X_{1}\right)=[-27.7 . .-1.5], \\
& \quad \text { overlapped_by_smallUrbArea }(X 1, X 2)=\text { true. }
\end{aligned}
$$

$C_{1}, C_{2}$ and $C_{3}$ cover adjacent areas with quite similar range value for deprivation indexes but $C_{1}$ models the presence of woods while $C_{2}$ and $C_{3}$ model the presence of small urban areas and large urban areas, respectively. Discontinuity of geographical environments modeled by these clusters is confirmed by visualizing map data about the area (see Figure 3).

The logical decision tree mined on the same data discovers 58 different clusters. Clusters are built by minimizing the distance among relational descriptions of wards. However, the discrete structure imposed by the adjacency relation is ignored. Hence, wards which are not connected in the graph imposed by the adjacency relation are clustered together.

\section{Conclusions}

This paper presents a novel approach to discover clusters from structured spatial data taking into account relational constraints (e.g. spatial correlation) forming the discrete spatial structure. We represent this discrete spatial structure as a graph such that the concept of graph neighborhood is exploited to capture relational constraints embedded in the graph edges. Moreover, we resort to a relational approach to mine data scattered in multiple relations describing the structure that is naturally embedded in spatial data. As a consequence, only spatial units associated with (transitively) graph connected nodes can be clustered together according to judgment of similarity on relational descriptions representing their internal (spatial) structure. As future work, we intend to integrate 
CORSO in a spatial data mining system that is able to extract both the spatial structure and the structure of spatial objects from a spatial database, cluster these spatial objects coherently with the extracted spatial structure and visualize discovered clusters. We also plan to employ CORSO for air pollution analysis.

\section{Acknowledgment}

The work presented in this paper is partial fulfillment of the research objective set by the ATENEO-2004 project on "Metodi di Data Mining Multi-relazionale per la scoperta di conoscenza in basi di dati".

\section{References}

1. L. De Raedt and H. Blockeel. Using logical decision trees for clustering. In S. Džeroski and N. Lavrač, editors, Inductive Logic Programming, 7th International Workshop, volume 1297, pages 133-140. Springer-Verlag, 1997.

2. S. Džeroski and N. Lavrač. Relational Data Mining. Springer-Verlag, 2001.

3. M. Egenhofer. Reasoning about binary topological relations. In Symposium on Large Spatial Databases, pages 143-160, 1991.

4. F. Esposito, D. Malerba, and G. Semeraro. Flexible matching for noisy structural descriptions. In International Joint Conference on Artificial Intelligence, pages 658-664, 1991.

5. M. Ester, H. P. Kriegel, and J. Sander. Algorithms and applications for spatial data mining. Geographic Data Mining and Knowledge Discovery, 5(6), 2001.

6. M. Ester, H.-P. Kriegel, J. Sander, and X. Xu. A density-based algorithm for discovering clusters in large spatial databases with noise. In Knowledge Discovery in Databases, pages 226-231, 1996.

7. V. Estivill-Castro and M. E. Houle. Robust distance-based clustering with applications to spatial data mining. Algorithmica, 30(2):216-242, 2001.

8. V. Estivill-Castro and I. Lee. Fast spatial clustering with different metrics and in the presence of obstacles. In International Symposium on Advances in geographic information systems, pages 142-147. ACM Press, 2001.

9. E. Hancock and M. Vento. Graph Based Representations in Pattern Recognitions. Springer-Verlag, 2003.

10. L. Holder and D. Cook. Graph-based relational learning: Current and future directions. SIGKDD Explorations Special Issues on Multi-Relational Data Mining, 5(1):90-93, 2003.

11. T. Horvath, S. Wrobel, and U. Bohnebeck. Relational instance-based learning with lists and terms. Machine Learning, 43(1/2):53-80, 2001.

12. L. Kaufmann and P. J. Rousseeuw. Finding Groups in Data: An Introduction to Cluster Analysis. John Wiley, 1990.

13. M. Kirsten and S. Wrobel. Relational distance-based clustering. In Inductive Logic Programming, 8th International Conference, volume 1446, pages 261-270. Springer-Verlag, 1998.

14. D. Malerba. Learning recursive theories in the normal ilp setting. Fundamenta Informaticae, 57(1):39-77, 2003. 
15. D. Malerba, F. Esposito, A. Lanza, F. A. Lisi, and A. Appice. Empowering a gis with inductive learning capabilities: The case of ingens. Journal of Computers, Environment and Urban Systems, Elsevier Science, 27:265-281, 2003.

16. D. Mavroeidis and P. Flach. Improved distances for structured data. In T. Horváth and A. Yamamoto, editors, Inductive Logic Programming, 13th International Conference, volume 2835, pages 251-268. Springer-Verlag, 2003.

17. A. M. Neville, J. and J. D. Clustering relational data using attribute and link information. In Text Mining and Link Analysis Workshop, 18th International Joint Conference on Artificial Intelligence, 2003.

18. R. T. Ng and J. Han. Efficient and effective clustering methods for spatial data mining. In J. Bocca, M. Jarke, and C. Zaniolo, editors, Very Large Data Bases, 20th International Conference, pages 144-155. Morgan Kaufmann Publishers, 1994.

19. D. Patterson. Introduction to Artificial Intelligence and expert systems. PrenticeHall, 1991.

20. L. D. Raedt and S. Dzeroski. First-order jk-clausal theories are pac-learnable. Artificial Intelligence, 70(1-2):375-392, 1994.

21. J. Sander, E. Martin, H.-P. Kriegel, and X. Xu. Density-based clustering in spatial databases: The algorithm gdbscan and its applications. Data Mining and Knowledge Discovery, 2(2):169-194, 1998.

22. W. Tobler. Cellular geography. In S. Gale and G.Olsson, editors, Philosophy in Geography, 1979.

23. G. Toussaint. Some unsolved problems on proximity graphs. In D. Dearholt and F. Harary, editors, First Workshop on Proximity Graphs, 1991.

24. M. Visvalingam. Operational definitions of area based social indicators. Environment and Planning, A(15):831-839, 1983.

\section{A Appendix}

Let us recall definitions (4) and (5) and apply them to numerical case. We have:

$$
f m(c,[a, b])=\max _{v \in[a, b]} P(\operatorname{equal}(c, v))=\max _{v \in[a, b]} P(\delta(X, v) \geq \delta(c, v))
$$

By assuming that $X$ has a uniform distribution on domain $D=[\alpha, \beta]$ with density function $f_{D}(x)=1 /(\beta-\alpha), \forall x \in D$ and fixing $\delta(x, y)=|x-y|$, $P(\delta(X, v) \geq \delta(c, v))$ can be rewritten as $P(|X-v| \geq|c-v|)$ that is maximized when minimizing $|c-v|$.

If $a \leq c \leq b$ then $\max _{v \in[a, b]} P(|X-v| \geq|c-v|)=P(|X-v| \geq|c-c|)=1$.

If $c<a$ then $\max _{v \in[a, b]} P(|X-v| \geq|c-v|)$ is written as $\max _{v \in[a, b]} P(|X-v| \geq v-c)$.

Since the maximum of $P(|X-v| \geq v-c)$ is obtained for $v=a$, we have that $\max _{v \in[a, b]} P(|X-v| \geq v-c)=P(|X-a| \geq a-c)=P(X-a \geq a-c)+P(X-a \leq$ $c-a)=P(X \geq 2 a-c)+P(X \leq c)$ where:

1. $P(X \geq 2 a-c)=\int_{\beta}^{2 a-c} 1 /(\beta-\alpha) d x=(\beta-2 a+c) /(\beta-\alpha)$ if $2 a-c \leq \beta, 0$ otherwise;

2. $P(X \leq c)=(c-\alpha) /(\beta-\alpha)$. 
Hence, we obtain that:

$\max _{v \in[a, b]} P(|X-v| \geq v-c)= \begin{cases}1-2(a-c) /(\beta-\alpha) & \text { if } c<a \wedge 2 a-c \leq \beta \\ (c-\alpha) /(\beta-\alpha) & \text { if } c<a \wedge 2 a-c>\beta\end{cases}$ If $c>b$ then $\max _{v \in[a, b]} P(|X-v| \geq|c-v|)$ can be equivalently written as $\max _{v \in[a, b]} P(\mid X$ $-v \mid \geq c-v)$ that is obtained for $v=b$. Therefore, $\max _{v \in[a, b]} P(|X-v| \geq c-v)=$ $P(|X-b| \geq c-b)=P(X-b \geq c-b)+P(X-b \leq b-c)=P(X \geq c)+P(X \leq$ $2 b-c)$. We have that:

$\max _{v \in[a, b]} P(|X-v| \geq c-v)= \begin{cases}(\beta-c) /(\beta-\alpha) & \text { if } c>b \wedge 2 b-c<\alpha \\ 1-2(c-b) /(\beta-\alpha) & \text { if } c>b \wedge 2 b-c \geq \alpha\end{cases}$ 